\title{
Exploring Topsnut-Graphical Passwords by Twin Odd-elegant Trees
}

\author{
Hong-yu WANG ${ }^{1}$, Jin $\mathrm{XU}^{1, \star}$, and Bing $\mathrm{Yao}^{2,3}$ \\ ${ }^{1}$ School of Electronics Engineering and Computer Science, Peking University, 100871, China \\ ${ }^{2}$ College of Mathematics and Statistics, Northwest Normal University, Lanzhou, 730070, China \\ ${ }^{3}$ College of Electronic and Information Engineering, Lanzhou Jiaotong University, Lanzhou 730070, China
}

\begin{abstract}
Graphical passwords are facing a good opportunity as 2-dimension codes are accepted by many people, since it has been applied in mobile devices, electronic equipments with touch screen, and so on. QR codes can be considered as a type of graphical passwords. Topsnut-graphical password differs from the existing graphical passwords, and has been investigated and developed. In this article, a new type of Topsnut-graphical passwords has been designed by technique of graph theory, called twin odd-elegant labelling. We make the twin odd-elegant graphs for one-key vs two or more locks (conversely, one-lock vs two or more keys). These Topsnut-GPWs show perfect matching characteristics of locks (TOE-lock-models) and keys (TOE-key-models). We show examples for testing our methods which can be easily transformed into effective algorithms.
\end{abstract}

\section{Introduction}

Since its introduction in 1994, the QR Code (Quick Response codes, QR-codes, they are referred as a printable computer language) has gained wide acceptance in such diverse industries as manufacturing, warehousing and logistics, retailing, healthcare, life sciences, transportation and office automation [2]. QR codes can be considered as a type of graphical passwords that are used widely, since it has been applied in mobile devices, electronic equipments with touch screen, and so on. QR code (abbreviated from Quick Response Code) is the trademark for a type of matrix barcode (or twodimensional barcode) first designed for the automotive industry in Japan. A barcode is a machine-readable optical label that contains information about the item to which it is attached. A QR code uses four standardized encoding modes (numeric, alphanumeric, byte/binary, and kanji) to efficiently store data; extensions may also be used [1]. However, QR codes can not be used to those departments required with highlevel security. We have known that passwords in the security community should be easy to remember, and the user authentication protocol should be executable quickly and easily by humans. And passwords should be secure, i.e. they should look random and should be hard to guess; they should be changed frequently, and should be different on different accounts of the same user; they should not be written down or stored in plain text. On the other hands, no report tells us that other graphical passwords were applied to business and were accepted by people (Ref. [7], [3], [5]). Wiedenbeck et al. [12] asked for two principal research questions:

\footnotetext{
^e-mail: jxu@pku.edu.cn
} 
Question 1: Are graphical passwords a viable alternative to alphanumeric passwords in terms of security, as well as password creation, learning, performance, and retention?

Question 2: Are users' perceptions of graphical passwords different from those of alphanumeric passwords?

An idea of "graphical structure plus number theory" (Topsnut) for creating new type of graphical passwords was proposed firstly by Wang et al. in [9, 10]. And in making new Topsnut graphical passwords (Topsnut-GPWs), Wang et al. [11] have designed some TopsnutGPWs by knowledge of graph theory for answering the following problem of number theory:

Problem of odd-graceful KL-matching pairs: Let $[0,2 q]=\{0,1,2, \ldots, 2 q\}$ and $[1,2 q-$ $1]^{o}=\{1,3,5, \ldots, 2 q-1\}$ with $q \geq 2$. If there are two subsets $X_{1}, X_{2}$ of $[0,2 q]$ hold: (i) $X_{1} \cup X_{2}=[0,2 q]$ and $\{0\} \subseteq X_{1} \cap X_{2} \subseteq[0,2 q]$; (2) $\left\{|a-b|: a \in X_{i}^{\text {odd }}, b \in X_{i}^{\text {even }}\right\}=[1,2 q-1]^{o}$ where $X_{i}^{\text {odd }}$ is an odd-number set and $X_{i}^{\text {even }}$ is an evennumber set of $X_{i}$ such that $X_{i}^{\text {odd }} \cup \in X_{i}^{\text {even }}=X_{i}$ and $X_{i}^{\text {odd }} \cap \in X_{i}^{\text {even }}=\emptyset$ with $i=1,2$. We say $X_{1}$ and $X_{2}$ to be an odd-graceful KL-matching pair. Find all odd-graceful KL-matching pairs for any given set $[0,2 q]$ with $q \geq 2$.

Clearly, one can use odd-graceful KLmatching pairs to design more complex Topsnut-GPWs. We, in Section 2, will introduce several techniques by knowledge of graph theory. Our main works are to show new TopsnutGPWs made by the techniques, which also are the part solutions of the problem of odd-elegant KL-matching pairs proposed in the last section. On the other hands, our methods for dealing with the new Topsnut-GPWs can be easily transformed into algorithms.

\section{Definitions for techniques}

We use standard notation and terminology of graph theory [4]. Graphs mentioned are loopless, no multiple edges, undirected, connected and finite, unless otherwise specified. A $(p, q)$ graph $G$ is one with $p$ vertices and $q$ edges. The shorthand symbol $[m, n]$ stands for an integer set $\{m, m+1, \ldots, n\}$, where $m$ and $n$ are integers with $0 \leq m<n$; the notation $[s, t]^{o}$ indicates an oddset $\{s, s+2, \ldots, t\}$, where $s$ and $t$ both are odd integers with $1 \leq s<t$; and the notation $[k, \ell]^{e}$ indicates an even-set $\{k, k+2, \ldots, \ell\}$, where $k$ and $\ell$ both are even integers with $0 \leq k<\ell$.

Definition 1 [14] Suppose that a $(p, q)$-graph $G$ admits a mapping $f: V(G) \rightarrow[0,2 q-1]$ such that $f(u) \neq f(v)$ for distinct $u, v \in V(G)$, and the label $f(u v)$ of every edge $u v \in E(G)$ is defined as $f(u v)=f(u)+f(v)(\bmod 2 q)$ and the set of all edge labels is equal to $[1,2 q-1]^{\circ}$, we call $f$ an odd-elegant labelling and $G$ to be odd-elegant.

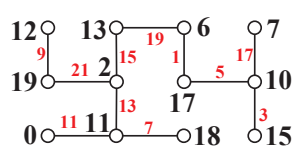

(a)

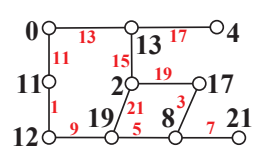

(b)
Figure 1. (a) An odd-elegant tree; (b) An odd-elegant graph.

Definition 2 [13] Suppose that a bipartite graph $G$ admits a labelling $f$ such that $\max \{f(x): x \in X\}<\min \{f(y): y \in Y\}$, where $(X, Y)$ is the bipartition of vertex set $V(G)$ of $G$. We call $f$ a set-ordered labelling (an Solabelling for short).

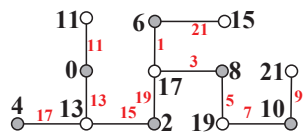

(a)

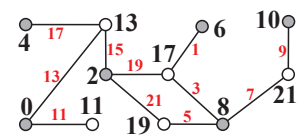

(b)
Figure 2. (a) A set-ordered odd-elegant tree; (b) A set-ordered odd-elegant graph.

Let $G_{j}$ be a $\left(p_{j}, q_{j}\right)$-graph with $j=1,2$. The graph $G$ obtained by identifying two certain vertices $x_{i, 1}$ of $G_{1}$ with two certain vertices $x_{i, 2}$ of $G_{2}$ into one vertex $x_{i}$, where $\mathrm{i}=1,2, x_{1}$ and $x_{2}$ is called the identified-vertex, respectively. is denoted as $G=G_{1} \circ_{2} G_{2}$, called an double identifying graph. So, the graph $G=G_{1} \circ_{2} G_{2}$ has $p_{1}+p_{2}-2$ vertices and $q_{1}+q_{2}$ edges.

Suppose that two vertices $w_{1}$ and $w_{2}$ of $G$ are two identified-vertices of identifying $x_{i, 1}$ with 
$x_{i, 2}$, where $i=1,2$. Then we can split the identified-vertices $w_{i}$ into two vertices $x_{i, 1}$ and $x_{i, 2}$ (called the splitting-vertices), so $G$ can be split into two parts $G_{1}$ and $G_{2}$.

The above process of producing $G$ from $G_{1}$ and $G_{2}$ is called an identifying operation, another process of splitting $G$ into two parts $G_{1}$ and $G_{2}$ is called a splitting operation.

Definition 3 Let two connected $\left(p_{i}, q\right)$-graphs $G_{i}$ with $i=1,2$, and let $p=p_{1}+p_{2}-2$. If the identifying $(p, q)$-graph $G=G_{1} \circ_{2} G_{2}$ has a mapping $f: V(G) \rightarrow[0, q-1]$ such that $(i)$ $f(x) \neq f(y)$ for any pair of vertices, $y \in V(G)$; (ii) $f$ is an odd-elegant labelling of $G_{i}$ with $i=1,2$. Then we say $G$ to be $a$ twin odd-elegant graph (a TOE-graph), $f$ a TOE-labelling. $G_{1}$ $a$ TOE-source graph, and $G_{2} a$ TOE-associated graph, vice versa.

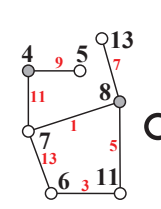

(a)

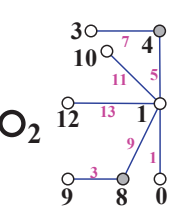

(b)

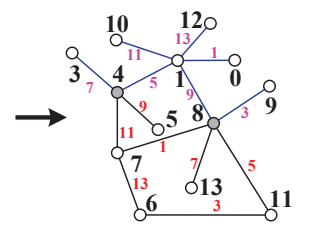

(c)
Figure 3. A twin odd-elegant graph $G=G_{1} \circ_{2} G_{2}$ with its OE-source graph $G_{1}$ and OE-associated graph $G_{2}$ by an identifying operation.

Furthermore, if each $G_{i}$ with $i=1,2$ is a connected graph in Definition 3, and exist TOE-source $G_{1}$ is a bipartite connected graph having its own bipartition $\left(X_{1}, Y_{1}\right)$ satisfy Definition 2 , we say that the identifying $(p, q)$ graph $G=G_{1} \circ_{2} G_{2}$ is a set-ordered twin oddelegant graph, $f$ a set-ordered twin odd-elegant labelling. Here, the source graph $G_{1}$ is a setordered odd-elegant graph by Definition 1 and 2.

Similarly, we present the definition of a twin odd-elegant tree as follows.

Definition 4 Let $T_{i}$ be a tree of order $n$ with $i=1,2$. If the identifying tree $G_{T}=T_{1} \circ_{2} T_{2}$ has a mapping $f: V\left(G_{T}\right) \rightarrow[0,2 n-3]$ such that $(i) f(x) \neq f(y)$ for any pair of vertices $x, y \in V\left(G_{T}\right)$; (ii) $f$ is an odd-elegant labelling of $T_{i}$, where $i=1,2$. Then $G_{T}$ is called $a$ twin odd-elegant tree (a TOE-tree), $f$ a TOElabelling, and $T_{1} a$ TOE-source tree, and $T_{2} a$ TOE-associated tree of $T_{1}$. Furthermore, we say $T_{1}$ a TOE-source self-associated tree if $T_{1}$ is isomorphic to $T_{2}$.

Two examples for illustrating Definition 4 are show in Figure 4 and Figure 10. Let $T_{1}$ has its bipartition $\left(X_{1}, Y_{1}\right)$ in Definition 2; and if the TOE-source tree $T_{1}$ has a set-ordered oddelegant labelling, then we call $G_{T}$ a set-ordered twin odd-elegant tree (an So-TOE tree for short), and $f$ is set-ordered twin odd-elegant labelling( an So-TOE labelling for short).

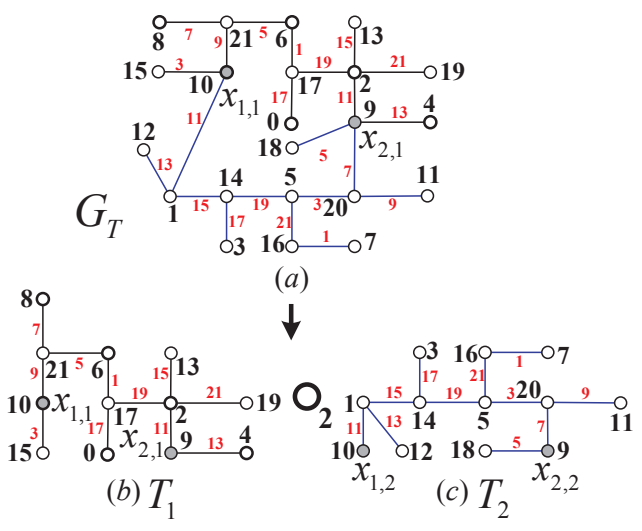

Figure 4. A TOE tree $G_{T}$ has its TOE-source tree $T_{1}$ and TOE-associated tree $T_{2}$, and two split-vertices $x_{i, 1}$ and $x_{i, 2}$ labelled with numbers 9 and 10 , where $i=1,2$, by a splitting operation.

In our design of the new Topsnut-GPWs, a source graph/tree and its associated graph/tree in Definition 3 or Definition 4 are called a TOElock-model and a TOE-key-model, respectively.

\section{Theoretical analysis and guarantee}

Lemma 1 Any star $K_{1, n}$ is a TOE-source tree of a So-TOE-tree $G_{T}$.

Proof. Let $V\left(K_{1, n}\right)=\left\{x, y_{1}, y_{2}, \ldots, y_{n}\right\}$ and $E\left(K_{1, n}\right)=\left\{x y_{i}: i \in[1, n]\right\}$ be the sets of vertices and edges of $K_{1, n}$, respectively. We can define an odd-elegant labelling $f$ with $f(x)=0$, 
$f\left(y_{i}\right)=2 i-1$ with $i \in[1, n]$, which induce $f\left(x y_{i}\right)=f(x)+f\left(y_{i}\right)(\bmod 2 n)$ for $i \in[1, n]$. So, $f\left(E\left(K_{1, n}\right)=[1,2 n-1]^{o}\right.$. i.e. $K_{1, n}=T_{1}$

Let $T_{2}$ be a copy of $K_{1, n}$. We have the sets of vertices and edges of $T_{2}$ as $V\left(T_{2}\right)=$ $\left\{x^{\prime}, y_{1}^{\prime}, y_{2}^{\prime}, \ldots, y_{n}^{\prime}\right\}$ and $E\left(T_{2}\right)=\left\{x^{\prime} y_{i}^{\prime}: i \in[1, n]\right\}$, respectively. Next, we define a labelling $f^{\prime}$ in the way $f^{\prime}\left(x^{\prime}\right)=1, f^{\prime}\left(y_{i}^{\prime}\right)=2 i-2$ with $i \in[1, n]$. Clearly, $f^{\prime}\left(x^{\prime} y_{i}^{\prime}\right)=f^{\prime}\left(x^{\prime}\right)+f^{\prime}\left(y_{i}^{\prime}\right)(\bmod 2 n)$ for $i \in[1, n]$. We have that $f^{\prime}\left(E\left(T_{2}\right)=[1,2 n-1]^{o}\right.$.

Since $f\left(y_{1}\right)=f^{\prime}\left(x^{\prime}\right)=1$ and $f(x)=$ $f^{\prime}\left(y_{1}^{\prime}\right)=0$, we identify the vertex $y_{1}$ of $K_{1, n}$ with the vertex $x^{\prime}$ of $T_{2}$ into one $w_{1}$ and the vertex $x$ of $K_{1, n}$ with the vertex $y_{1}^{\prime}$ of $T_{2}$ into one $w_{2}$ for forming the identifying tree $G_{T}=$ $K_{1, n} \circ_{2} T_{2}$. It is not hard to provide a labelling $f_{G_{T}}$ of $G_{T}$ as: $f_{G_{T}}(u)=f(u)$ for $u \in V\left(K_{1, n}\right)$, and $f_{G_{T}}(v)=f^{\prime}(v)$ for $v \in V\left(T_{2}\right)$. Immediately, $\left\{f\left(x y_{i}\right): x y_{i} \in E\left(K_{1, n}\right)\right\}=[1,2 n-1]^{o}$ and $\left\{f\left(x^{\prime} y_{i}^{\prime}\right): x^{\prime} y_{i}^{\prime} \in E\left(T_{2}\right)\right\}=[1,2 n-1]^{o}$. Therefor, we claim that $G_{T}$ is a TOE-tree since $f_{G_{T}}$ is a TOE-labelling of $G_{T}$ by Definition 4, which means that $K_{1, n}$ is an So-TOE source tree of $G_{T}$, and $T_{2}$ is a TOE-associated tree of $K_{1, n}$.

It is noticeable, $T_{2}=K_{1, n}$ means that the TOE-source tree $K_{1, n}$ is isomorphic to its associated tree $T_{2}$ in $T$. So, $K_{1, n}$ is a TOE-source self-associated tree.

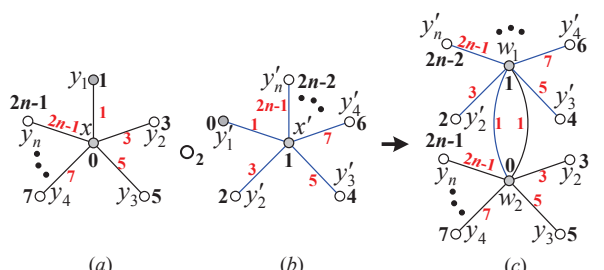

(a)

(b)

(c)

Figure 5. An illustration for Lemma 1.

Theorem 1 Any set-ordered odd-elegant tree being not a star is an So-TOE-source tree of at least two So-TOE trees.

Proof. Suppose that $T_{1}$ is a tree with $n$ vertices, its vertex bipartition is $(X, Y)$, where $X=\left\{x_{i}: i \in[1, s]\right\}, Y=\left\{y_{j}: j \in[1, t]\right\}$, $s+t=n$ and $\min \{s, t\} \geq 2$. By the hypothesis of the theorem, $T_{1}$ has a set-ordered oddelegant labelling $f_{1}$ defined by $f_{1}\left(x_{i}\right)=2(i-1)$, $i \in[1, s] ; f_{1}\left(y_{j}\right)=2(j+s-1)-1, i \in[1, t] ;$ and $f_{1}\left(x_{i} y_{j}\right)=f_{1}\left(y_{j}\right)+f_{1}\left(x_{i}\right)=2(s+i+j-2)-1$ $(\bmod 2(n-1))$ for each edge $x_{i} y_{j} \in E\left(T_{1}\right)$.

We can observe $f_{1}\left(V\left(T_{1}\right)\right)=\{0,2, \ldots, 2 s-$ $2,2 s-1,2 s+1, \ldots, 2 n-3\}$ and $f_{1}\left(E\left(T_{1}\right)\right)=$ $[1,2 n-3]^{\circ}$.

Case 1. We construct a labelling $f_{2}$ of another tree $T_{2}$ by the labelling $f_{1}$ such that $f_{2}\left(V\left(T_{2}\right)\right)=\{1,3, \ldots, 2 s-3,2 s-2,2 s-$ $1,2 s, \ldots, 2(n-2)\}$, and $f_{2}\left(E\left(T_{2}\right)\right)=\left\{f_{2}(u v)=\right.$ $\left.f_{2}(u)+f_{2}(v)(\bmod 2(n-1)): u v \in E\left(T_{2}\right)\right\}=$ $[1,2 n-3]^{o}$, and furthermore, $f_{2}(u) \neq f_{2}(v)$ for any $u, v \in V\left(T_{2}\right)$. This tree $T_{2}$ can be built up in the way: A bipartition $\left(U_{1}, V_{1}\right)$ with $U_{1}=\left\{u_{i}\right.$ : $i \in[1, s]\}$ and $V_{1}=\left\{v_{j}: j \in[1, t]\right\}$, such that $f_{2}\left(u_{i}\right)=2 i-1, i \in[1, s] ; f_{2}\left(v_{j}\right)=2(s-2+j)$, $j \in[1, t]$. Any edge $u_{i} v_{j} \in E\left(T_{2}\right)$ satisfy $f_{2}\left(u_{i} v_{j}\right)=f_{2}\left(u_{i}\right)+f_{2}\left(v_{j}\right)(\bmod 2(n-1))$ with $i \in[1, s]$ and $j \in[1, t]$. We construct the edge set of $T_{2}$ as $\left\{u_{1} v_{j}, u_{i} v_{t}: i \in[1, s], j \in[1, t-1]\right\}$ such that the edge labels are $f_{2}\left(u_{i} v_{t}\right)=2 i-3$, $f_{2}\left(u_{1} v_{j}\right)=2 j+2 s-3(\bmod 2(n-1))$ for $i \in[1, s]$ and $j \in[1, t-1]$. Observe that $f_{2}\left(E\left(T_{2}\right)\right)=$ $[1,2 n-3]^{o}, f_{1}\left(y_{1}\right)=f_{2}\left(u_{s}\right)$ and $f_{1}\left(x_{s}\right)=f_{2}\left(v_{1}\right)$.

Now, we can identify the vertex $x_{s}$ and $y_{1}$ of $T_{1}$ with the vertex $v_{1}$ and $u_{s}$ of $T_{2}$ into one (the identified vertex) $w_{1}$ and $w_{2}$, respectively, so we obtain the desired tree $G_{T}=T_{1} \circ_{2} T_{2}$. And $G_{T}$ has a labelling $f$ defined as: $f\left(x_{i}\right)=$ $f_{1}\left(x_{i}\right), i \in[1, s-1] ; f\left(y_{j}\right)=f_{1}\left(y_{j}\right), i \in[2, t]$; $f\left(u_{k}\right)=f_{2}\left(u_{k}\right), k \in[1, s-1], f\left(v_{l}\right)=f_{2}\left(v_{l}\right)$, $l \in[2, t], f\left(w_{1}\right)=2 s-2$ and $f\left(w_{2}\right)=2 s-1$. Clearly, any two vertices of $G_{T}$ are assigned different numbers. According to Definition $4, G_{T}$ is an So TOE-tree having the source tree $T_{1}$.

Case 2. Similarly to Case 1, we can get the following results: Let $f_{2}\left(V\left(T_{2}^{\prime}\right)\right)=\{1,3, \ldots, 2 s-$ $3,2 s-2,2 s, 2(s+1), \ldots, 2 n-4\} \cup\{0\}$, and $f_{2}\left(E\left(T_{2}^{\prime}\right)\right)=[1,2 n-3]^{\circ}$, and furthermore $f_{2}(u) \neq$ $f_{2}(v)$ for $u, v \in V\left(T_{2}^{\prime}\right)$. This tree $T_{2}^{\prime}$ can be built up in the way: A bipartition $\left(U_{2}, V_{2}\right)$ with $U_{2}=\left\{u_{i}: i \in[1, s-1]\right\}$ and $V_{2}=\left\{v_{j}:\right.$ $j \in[1, t+1]\}$, such that $f_{2}\left(u_{i}\right)=2 i-1$, $i \in[1, s-1] ; f_{2}\left(v_{j}\right)=2(s-2+j), j \in[1, t]$, $f_{2}\left(v_{t+1}\right)=0$. Any edge $u_{i} v_{j} \in E\left(T_{2}^{\prime}\right)$ satisfies $f_{2}\left(u_{i} v_{j}\right)=f_{2}\left(u_{i}\right)+f_{2}\left(v_{j}\right)(\bmod 2(n-1))$ with $i \in$ [1,s-1] and $j \in[1, t+1]$. We construct the edge set of $T_{2}^{\prime}$ as $\left\{u_{1} v_{j}, u_{i} v_{t+1}: i \in[2, s], j \in[1, t]\right\}$ 
such that the edge labels are $f_{2}\left(u_{i} v_{t+1}\right)=2 i-1$, $f_{2}\left(u_{1} v_{j}\right)=2(s+j)-3$ for $i \in[1, s-1]$ and $j \in[1, t]$. Observe that $f_{2}\left(E\left(T_{2}^{\prime}\right)\right)=[1,2 n-3]^{o}$, $f_{1}\left(x_{1}\right)=f_{2}\left(v_{t+1}\right)$ and $f_{1}\left(x_{s}\right)=f_{2}\left(v_{1}\right)$.

Now, we can identify the vertex $x_{1}$ and $x_{s}$ of $T_{1}$ with the vertex $v_{t+1}$ and $v_{1}$ of $T_{2}^{\prime}$ into one (the identified vertex) $w_{1}$ and $w_{2}$, so we obtain the desired tree $G_{T^{\prime}}=T_{1} \circ_{2} T_{2}^{\prime}$. And $G_{T^{\prime}}$ has a labelling $f$ defined as: $f\left(x_{i}\right)=f_{1}\left(x_{i}\right), i \in[2, s-1]$; $f\left(y_{j}\right)=f_{1}\left(y_{j}\right), i \in[1, t] ; f\left(u_{k}\right)=f_{2}\left(u_{k}\right)$, $k \in[1, s-1], f\left(v_{l}\right)=f_{2}\left(v_{l}\right), l \in[2, t], f\left(w_{1}\right)=0$ and $f\left(w_{2}\right)=2 s-2$. Clearly, any two vertices of $G_{T^{\prime}}$ are assigned different numbers. According to Definition $4, G_{T^{\prime}}$ is an So-TOE tree having the source tree $T_{1}$.

Corollary 1 (1) Every So-TOE-source tree is a TOE-source self-associated tree.

(2) Not every TOE-tree consisting of TOEsource self-associated tree has an odd-elegant labelling.

Theorem 2 If the TOE-source tree $T_{1}$ is a setordered odd-elegant tree, then the simple SoTOE graph $G_{T}=T_{1} \circ_{2} T_{2}$ has an odd-elegant labelling.

\section{Test and simultaneous}

Experiment 1 We show two examples for illustrating the results of Theorem 1. Our methods appeared in the proof of Theorem 1 are easily applied the design of Topsnut-GPws, since the methods are constructive. We illustrate theorem 2 with Figure 6(c) and Figure 9.

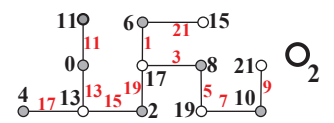

(a)

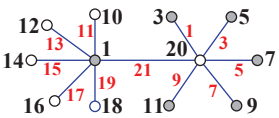

(b)

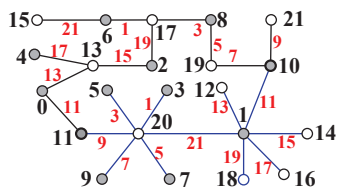

(c)

Figure 6. An example for illustrating Case 1 of Theorem 1.

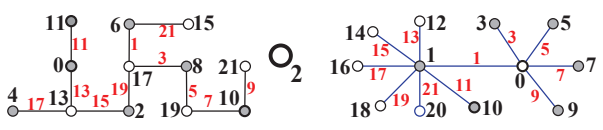

(a)

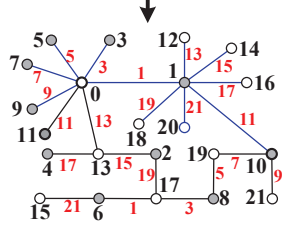

(c)

Figure 7. An example for illustrating Case 2 of Theorem 1.

Experiment 2 Based on the technique provided by Corollary 1, we make a Topsnut-GPW shown in Figure 8(c) for the purpose of simultaneous.

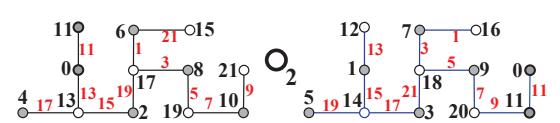

(a)

(b)

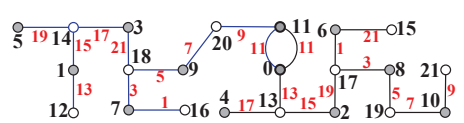

(c)

Figure 8. An example for illustrating the technique of Corollary 1.

Experiment 3 It is is very important to get the property $P$ of large scale of topological structure for smaller topological structures having a property $P$. We test the results of Theorem 2 by the scheme shown in Figure 9. Here, we can confirm that our method for proving Theorem 2 can be transformed into an effective algorithm.

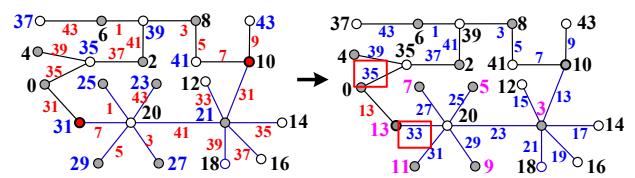

(a)

(b)

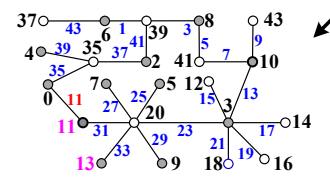

(c) An odd-elegant graph 
Figure 9. A conversion process from an So-TOE-tree to odd-elegant graph for illustrating Theorem 2.

Experiment 4 We have the following results (1) A TOE-source graphs may have different TOEassociated graphs, and vice versa.

(2) A TOE-graph may contain two or more groups of TOE-source and TOE-associated graphs.

(3) Two groups of TOE-source and TOEassociated graphs may produce a TOE-graph.

As shown in Figure 11, there are eight different TOE-key-models for Figure $10(\mathrm{~b})$.

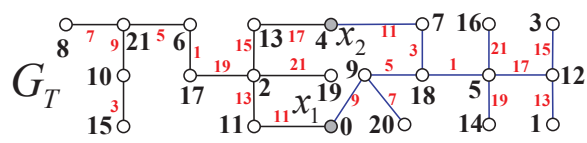

(a)

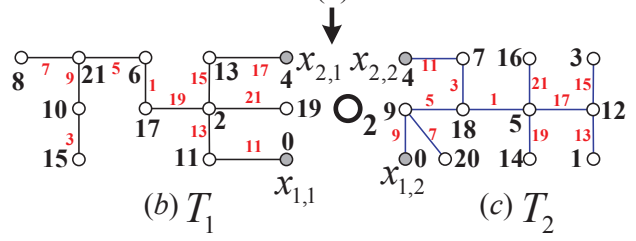

Figure 10. An So-TOE tree $G_{T}$ has its TOE-source tree $T_{1}$ and TOE-associated tree $T_{2}$, and two splitting-vertices $x_{i, 1}$ and $x_{i, 2}$ labelled with numbers 0 and 4 , where $i=1,2$, by a splitting operation.

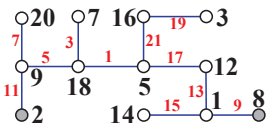

(a)

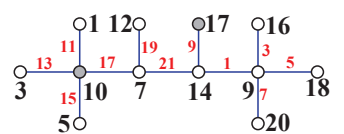

(c)



(e)



$(g)$

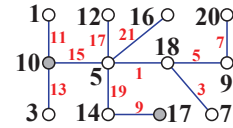

(b)

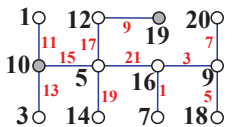

(d)

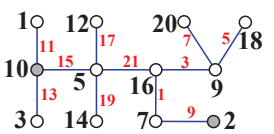

$(f)$

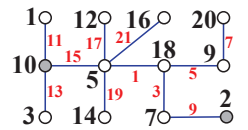

(h)
Figure 11.(a)-(h) Eight TOE-key-models of the TOE-lock-model for Figure 10(b) (eight TOE-associated trees of the TOE-source tree $T_{1}$ ).

\section{Conclusion and researching problems}

We have designed a new technique, called (setordered) twin odd-elegant labelling, by the idea of graph labellings. Furthermore, we have found some themods/algorithms to realize TopsnutGPWs based our new graph labellings. These Topsnut-GPWs show perfect matching characteristics of locks (TOE-lock-models) and keys (TOE-key-models), which enable us to produce one-key vs two or more locks (conversely, onelock vs two or more keys). We have many graph colorings were introduced and investigated by algorithmic methods [4], and have more graph labellings shown in a large survey due to Gallian [6], the survey collected over 1400 papers. Thereby, Topsnut-GPWs are supported by graph colorings/labellings. Every thing can become a password, and $\mathrm{t}$ is just beginning for TopsnutGPWs. By our experience, we propose:

Conjecture 1 Any tree is the TOE-source tree of a certain twin odd-elegant tree.

Conjecture 2 Any twin odd-elegant tree is simple having an odd-elegant labelling.

Furthermore, we, motivated from problem of odd-graceful KL-matching pairs, propose the following problem of number theory:

Odd-elegant KL-matching pairs' problem. Let $[0,2 q-1]=\{0,1,2, \ldots, 2 q-1\}$ and $[1,2 q-1]^{o}=\{1,3,5, \ldots, 2 q-1\}$ with integer $q \geq 2$. If there are two subsets $Y_{1}$ and $Y_{2}$ of $[0,2 q-1]$ such that

(I) $Y_{1} \cup Y_{2}=[0,2 q-1]$ and $\{0, k\}=Y_{1} \cap Y_{2} \subseteq$ [0,2q-1] for some $k \in[1,2 q-1]$; and

(II) the set $\left\{a+b(\bmod 2 q): a \in Y_{i}^{\text {odd }}, b \in\right.$ $\left.Y_{i}^{\text {even }}\right\}=[1,2 q-1]^{o}$, where $Y_{i}^{\text {odd }}$ is an oddnumber set and $Y_{i}^{\text {even }}$ is an even-number set of $Y_{i}$ holding $Y_{i}^{\text {odd }} \cup Y_{i}^{\text {even }}=Y_{i}$ and $Y_{i}^{\text {odd }} \cap Y_{i}^{\text {even }}=\emptyset$ with $i=1,2$. 
We call these two sets $Y_{1}$ and $Y_{2}$ to be an odd-elegant KL-matching pair, and want to find all possible odd-elegant KL-matching pairs.

\section{Acknowledgment}

This work is supported by National Key R\&D Program of China (No. 2016YFB0800700), National 973 Program of China (2013CB329600) and the National Natural Science Foundation of China (Nos. 61572046, 61502012, 61672050, 61363060 and 61662066).

\section{References}

[1] "QR Code features". Denso-Wave. Archived from the original on 2013-01-29. Retrieved 3 October 2011.

[2] "QR Code Essentials". Denso ADC. 2011. Retrieved 12 March 2013.

[3] R. Biddle, S. Chiasson, P. C. V. Oorschot. Graphical passwords:Learning from the First Twelve Years. Acm Computing Surveys, 44,4 (2012):1-41.

[4] J. A. Bondy, U. S. R. Murty. Graph Theory. Springer London, 2008.

[5] H. Gao, W. Jia, F. Ye, et al. A survey on the use of graphical passwords in security . Journal of Software, 8, 7(2013):320-329.

[6] J. A. Gallian. A Dynamic Survey of Graph Labeling. The electronic journal of combinatorics, 17 (2014), \# DS6.

[7] X. Suo, Y. Zhu , G. S. Owen. Graphical Passwords: A Survey. Computer Security
Applications Conference. DBLP, (2005):463472.

[8] X. Suo . A Study of Graphical Password for Mobile Devices. International Conference on Mobile Computing, Applications, and Services. Springer, Cham, (2013): 202-214.

[9] H. Y. Wang, J. Xu , B. Yao. Exploring New Cryptographical Construction of Complex Network Data. IEEE International Conference on Data Science in Cyberspace. IEEE, (2016):155-160.

[10] H. Y. Wang, J. Xu , B. Yao. The keymodels and their lock-models for designing new labellings of networks. Advanced Information Management, Communicates, Electronic and Automation Control Conference. IEEE,(2016):565-568.

[11] H. Y. Wang, J. Xu , B. Yao. Twin Oddgraceful Trees Towards Information Security. Procedia Computer Science 107 (2017):1520, Elsevier Science Publishers B. V. 2017, DOI: 10.1016/j.procs.2017.03.050

[12] S. Wiedenbeck, J. Waters , J. C. Birget, et al. PassPoints: design and longitudinal evaluation of a graphical password system[J]. International Journal of HumanComputer Studies, 63, (1-2)(2005):102-127.

[13] B. Yao, H. Cheng, M. Yao, et al. A note on strongly graceful trees. Ars Combinatoria, 92 (2009):155-169.

[14] X. Q. Zhou, B. Yao, X. E. Chen. Every lobster is odd-elegant. Information Processing Letters, 113,1-2(2013):30-33. 